\title{
Some new results for single-valued and multi-valued mixed monotone operators of Rhoades type
}

Farshid Khojasteh ${ }^{*}$

${ }^{*}$ Correspondence:

f-khojaste@iau-arak.ac.ir Department of Mathematics, Arak Branch, Islamic Azad University, Arak, Iran

\begin{abstract}
In (2008), Zhang proved the existence of fixed points of mixed monotone operators along with certain convexity and concavity conditions. In this paper, mixed monotone single-valued and multi-valued operators of Rhoades type are defined and two fixed point theorems are proved.

MSC: $47 \mathrm{H} 10 ; 47 \mathrm{HO}$

Keywords: mixed monotone operator; Rhoades type; multi-valued; increasing inward mappings; $\mathcal{L}^{\prime \prime}$-function
\end{abstract}

\section{Introduction and preliminaries}

In (1987), mixed monotone operators were introduced by Guo and Lakshmikantham [1]. Then many authors studied them in Banach spaces and obtained lots of interesting results (see $[2,3]$ and $[4-8]$ ).

On the other hand, in (2001), Rhoades [9] introduced a new fixed point theorem as a generalization of Banach fixed point theorem.

Theorem 1.1 (Rhoades [9]) Let $(X, d)$ be a complete metric space. Suppose that $T: X \rightarrow X$ is a single-valued mapping that satisfies

$$
d(T x, T y) \leq d(x, y)-\psi(d(x, y))
$$

for each $x, y \in X$, where $\psi:[0,+\infty) \rightarrow[0,+\infty)$ is continuous, nondecreasing and $\psi^{-1}(0)=$ $\{0\}$ (i.e., weakly contractive mappings). Then $T$ has a fixed point.

In this paper, a weak mixed monotone single-valued and multi-valued operator of Rhoades type is defined. Then two fixed point theorems for this kind of operators are proved.

Let $E$ be a real Banach space. The zero element of $E$ is denoted by $\theta$. A subset $P$ of $E$ is called a cone if and only if:

- $P$ is closed, nonempty and $P \neq\{\theta\}$,

- $a, b \in \mathrm{R}, a, b \geq 0$ and $x, y \in P$ imply that $a x+b y \in P$,

- $x \in P$ and $-x \in P$ imply that $x=\theta$.

o 2013 Khojasteh; licensee Springer. This is an Open Access article distributed under the terms of the Creative Commons Attribution License (http://creativecommons.org/licenses/by/2.0), which permits unrestricted use, distribution, and reproduction in any medium, provided the original work is properly cited. 
Given a cone $P \subset E$, a partial ordering $\leq$ with respect to $P$ is defined by $x \leq y$ if and only if $y-x \in P$. We write $x<y$ to indicate that $x \leq y$ but $x \neq y$, while $x \ll y$ stands for $y-x \in \operatorname{int} P$, where int $P$ denotes the interior of $P$. The cone $P$ is called normal if there exists a number $K>0$ such that $\theta \leq x \leq y$ implies $\|x\| \leq K\|y\|$ for every $x, y \in E$. The least positive number satisfying this is called the normal constant of $P$.

Assume that $u_{0}, v_{0} \in E$ and $u_{0} \leq v_{0}$. The set $\left\{x \in E: u_{0} \leq x \leq v_{0}\right\}$ is denoted by $\left[u_{0}, v_{0}\right]$. Now, we recall the following definitions from $[2,3]$.

Definition 1.1 Let $P$ be a cone of a real Banach space $E$. Suppose that $D \subset P$ and $\alpha \in$ $(-\infty,+\infty)$. An operator $A: D \rightarrow D$ is said to be $\alpha$-convex ( $\alpha$-concave) if it satisfies $A(t x) \leq$ $t^{\alpha} A x\left(A(t x) \geq t^{\alpha} A x\right)$ for $(t, x) \in(0,1) \times D$.

Definition 1.2 Let $E$ be an ordered Banach space and $D \subset E$. An operator is called mixed monotone on $D \times D$ if $A: D \times D \rightarrow E$ and $A\left(x_{1}, y_{1}\right) \leq A\left(x_{2}, y_{2}\right)$ for any $x_{1}, x_{2}, y_{1}, y_{2} \in D$, where $x_{1} \leq x_{2}$ and $y_{2} \geq y_{1}$. Also, $x^{*} \in D$ is called a fixed point of $A$ if $A\left(x^{*}, x^{*}\right)=x^{*}$.

Let $\mathcal{C}(E)$ be a collection of all closed subsets of $E$.

Definition 1.3 For two subsets $X, Y$ of $E$, we write

- $X \preceq Y$ if for all $x \in X$, there exists $y \in Y$ such that $x \leq y$,

- $x \prec X$ if there exists $z \in X$ such that $x \ll z$,

- $X \prec x$ if for all $z \in X, z \ll x$.

Definition 1.4 Let $D$ be a nonempty subset of $E$. $T: D \rightarrow \mathcal{C}(E)$ is called increasing (decreasing) upward if $u, v \in D, u \leq v$ and $x \in T(u)$ imply there exists $y \in T(v)$ such that $x \leq y$ $(x \geq y)$. Similarly, $T: D \rightarrow \mathcal{C}(E)$ is called increasing (decreasing) downward if $u, v \in D$, $u \leq v$ and $y \in T(v)$ imply there exists $x \in T(u)$ such that $x \leq y(x \geq y)$. T is called increasing (decreasing) if $T$ is an increasing (decreasing) upward and downward.

Definition 1.5 Let $D$ be a nonempty subset of $E$. A multi-valued operator $T: D \times D \rightarrow$ $\mathcal{C}(E)$ is said to be mixed monotone upward if $T(x, y)$ is increasing upward in $x$ and decreasing upward in $y$, i.e.,

$\left(\mathrm{A}_{1}\right)$ for each $y \in D$ and any $x_{1}, x_{2} \in D$ with $x_{1} \leq x_{2}$, if $u_{1} \in T\left(x_{1}, y\right)$, then there exists a $u_{2} \in T\left(x_{2}, y\right)$ such that $u_{1} \leq u_{2}$;

$\left(\mathrm{A}_{2}\right)$ for each $x \in D$ and any $y_{1}, y_{2} \in D$ with $y_{1} \leq y_{2}$, if $v_{1} \in T\left(x, y_{1}\right)$, then there exists a $v_{2} \in T\left(x, y_{2}\right)$ such that $v_{1} \geq v_{2}$.

Definition $1.6 x^{*} \in D$ is called a fixed point of $T$ if $x^{*} \in T\left(x^{*}, x^{*}\right)$.

Definition 1.7 [10] A function $\Psi:[0,1) \times P \times P \times E \rightarrow E$ is called an $\mathcal{L}^{\prime \prime}$-function if $\Psi(t, x, y, 0)=0, \Psi(t, x, y, s) \gg 0$ for $s \gg 0$, and $\Psi(t, x, y, z)<z$ for all $(t, x, y, z) \in[0,1) \times$ $P \times P \times E$.

In 2011, Khojasteh and Razani [10] extended the results given by Zhang [6]. Also, in 2011 Khojasteh and Razani [11] introduced the concept of integral with respect to a cone. We recall the following definitions and lemmas of cone integration and refer to [11, 12] for their proofs. 
Definition 1.8 [11] Suppose that $P$ is a cone in $E$. Let $a, b \in E$ and $a<b$. Define

$$
[a, b]:=\{x \in E: x=t b+(1-t) a \text { for some } t \in[0,1]\}
$$

and

$$
[a, b):=\{x \in E: x=t b+(1-t) a \text { for some } t \in[0,1)\} .
$$

Definition 1.9 [11] The set $\left\{a=x_{0}, x_{1}, \ldots, x_{n}=b\right\}$ is called a partition for $[a, b]$ if and only if the intervals $\left\{\left[x_{i-1}, x_{i}\right)\right\}_{i=1}^{n}$ are pairwise disjoint and $[a, b]=\left\{\bigcup_{i=1}^{n}\left[x_{i-1}, x_{i}\right)\right\} \cup\{b\}$. Denote $\mathcal{P}[a, b]$ as the collection of all partitions of $[a, b]$.

Definition 1.10 [12] For each partition $Q$ of $[a, b]$ and each increasing function $\phi$ : $[a, b] \rightarrow E$, we define cone lower summation and cone upper summation as

$$
L_{n}^{\mathrm{Con}}(\phi, Q)=\sum_{i=0}^{n-1} \phi\left(x_{i}\right)\left\|x_{i}-x_{i+1}\right\|
$$

and

$$
U_{n}^{\mathrm{Con}}(\phi, Q)=\sum_{i=0}^{n-1} \phi\left(x_{i+1}\right)\left\|x_{i}-x_{i+1}\right\|
$$

respectively. Also, we denote $\|\Delta(Q)\|=\sup \left\{\left\|x_{i}-x_{i-1}\right\|, x_{i} \in Q\right\}$.

Definition 1.11 [12] Suppose that $P$ is a cone in $E . \phi:[a, b] \rightarrow E$ is called an integrable function on $[a, b]$ with respect to a cone $P$ or, to put it simply, a cone integrable function if and only if for all partition $Q$ of $[a, b]$,

$$
\lim _{\|\Delta(Q)\| \rightarrow 0} L_{n}^{\mathrm{Con}}(\phi, Q)=S^{\mathrm{Con}}=\lim _{\|\Delta(Q)\| \rightarrow 0} U_{n}^{\mathrm{Con}}(\phi, Q),
$$

where $S^{\text {Con }}$ must be unique.

We show the common value $S^{\text {Con }}$ by

$$
\int_{a}^{b} \phi(x) d_{P}(x) \text { or to simplicity } \int_{a}^{b} \phi d_{p}
$$

We denote the set of all cone integrable functions $\phi:[a, b] \rightarrow E$ by $\mathcal{L}^{1}([a, b], E)$.

Lemma 1.1 [11] Let $M$ be a subset of $P$. The following conditions hold:

(1) If $[a, b] \subseteq[a, c] \subset M$, then $\int_{a}^{b} f d_{p} \leq \int_{a}^{c} f d_{p}$ for $f \in \mathcal{L}^{1}(M, P)$.

(2) $\int_{a}^{b}(\alpha f+\beta g) d_{p}=\alpha \int_{a}^{b} f d_{p}+\beta \int_{a}^{b} g d_{p}$ for $f, g \in \mathcal{L}^{1}(M, P)$ and $\alpha, \beta \in \mathrm{R}$.

Remark 1.1 [13, Remark 1.2] Let $P$ be a cone of $E$, and let $u \in P$. If for each $\epsilon \in \operatorname{int}(P)$, $0 \leq u \ll \epsilon$, then $u=0$. 


\section{Main results}

In this section, we introduce some new fixed point theorems in the class of mixed monotone operators. Due to this, the following definition is presented.

Definition 2.1 A mixed monotone operator $A: D \times D \rightarrow E$ is said to be a Weak Mixed Monotone single-valued operator of Rhoades type ( $\mathrm{WM}_{2} \mathrm{R}$ property for short) if

$$
A(t x, y) \leq A(x, t y)-\Psi(t, x, y, A(x, t y))
$$

for all $(x, y) \in D \times D$, where $\Psi:[0,1) \times P \times P \times E \rightarrow E$ is an $\mathcal{L}^{\prime \prime}$-function.

Theorem 2.1 Let $P$ be a cone of $E$, let $S$ be a completely ordered closed subset of $E$ with $S_{0}=S \backslash\{\theta\} \subset$ int $P$ and let $\lambda S \subset S$ for all $\lambda \in[0,1]$. Let $u_{0}, v_{0} \in S_{0}, A: P \times P \rightarrow E$ be a weak mixed monotone operator of Rhoades type with $A\left(\left(\left[\theta, v_{0}\right] \cap S\right) \times\left(\left[\theta, v_{0}\right] \cap S\right)\right) \subset S$ satisfying the following conditions:

(I) there exists $r_{0}>0$ such that $u_{0} \geq r_{0} v_{0}$,

(II) $A\left(u_{0}, v_{0}\right) \ll u_{0} \ll v_{0} \ll A\left(v_{0}, u_{0}\right)$,

(III) for $u, v \in\left[u_{0}, v_{0}\right] \cap S$ with $A(u, v) \ll u \ll v$, there exists $u^{\prime} \in S$ such that $u \leq A\left(u^{\prime}, v\right) \ll u^{\prime} \ll v$; similarly, for $u, v \in\left[u_{0}, v_{0}\right] \cap S$ with $u \ll v \ll A(v, u)$, there exists $v^{\prime} \in S$ such that $u \ll v^{\prime} \ll A\left(v^{\prime}, u\right) \leq v$.

Then $A$ has at least one fixed point $x^{*} \in\left[u_{0}, v_{0}\right] \cap S$.

Proof By the above condition (III), there exists $u_{1} \in S$ such that $u_{0} \leq A\left(u_{1}, v_{0}\right) \ll u_{1} \ll$ $v_{0}$. Then there exists $v_{1} \in S$ such that $u_{1} \ll v_{1} \ll A\left(v_{1}, u_{1}\right) \leq v_{0}$. Likewise, there exists $u_{2} \in S$ such that $u_{1} \leq A\left(u_{2}, v_{1}\right) \ll u_{2} \ll v_{1}$. Then there exists $v_{2} \in S$ such that $u_{2} \ll v_{2} \ll$ $A\left(v_{2}, u_{2}\right) \leq v_{1}$. In general, there exists $u_{n} \in S$ such that $u_{n-1} \leq A\left(u_{n}, v_{n-1}\right) \ll u_{n} \ll v_{n-1}$. Then there exists $v_{n} \in S$ such that $u_{n} \ll v_{n} \ll A\left(v_{n}, u_{n}\right) \leq v_{n-1}(n=1,2, \ldots)$.

Take $r_{n}=\sup \left\{r \in(0,1): u_{n} \geq r v_{n}\right\}$, thus $0<r_{0}<r_{1}<\cdots<r_{n}<r_{n+1}<\cdots<1$ and $\lim _{n \rightarrow \infty} r_{n}=\sup \left\{r_{n}: n=0,1,2, \ldots\right\}=r^{*} \in(0,1]$. Since $r_{n+1}>r_{n}=\sup \left\{r \in(0,1): u_{n} \geq r v_{n}\right\}$, thus $u_{n} \nsucceq r_{n+1} v_{n}$. In addition, $S$ is completely ordered and $\lambda S \subset S$ for all $\lambda \in[0,1]$, then $u_{n}<r_{n+1} v_{n}$. Now, one can prove $r^{*}=1$. Otherwise, $r^{*} \in(0,1)$.

Since $u_{n}<r_{n+1} v_{n}$ and $r_{n+1}<r^{*}$, hence $u_{n}<r^{*} v_{n}$, and we have

$$
\begin{aligned}
A\left(u_{n+1}, v_{n+1}\right) & \leq A\left(\frac{1}{r^{*}} u_{n+1}, r^{*} v_{n+1}\right) \\
& \leq A\left(u_{n+1}, v_{n+1}\right)-\Psi\left(r^{*}, \frac{1}{r^{*}} u_{n+1}, v_{n+1}, A\left(u_{n+1}, v_{n+1}\right)\right) \\
& <A\left(u_{n+1}, v_{n+1}\right),
\end{aligned}
$$

which is a contradiction. Thus, $r^{*}=1$. Let $\epsilon \gg 0$ be given. Choose $\delta>0$ such that $\epsilon+$ $N_{\delta}(0) \subseteq P$, where $N_{\delta}(0)=\{y \in E:\|y\|<\delta\}$. Since $r_{n} \rightarrow 1$, one can choose a natural number $N_{1}$ such that $\left(1-r_{n}\right) v_{1} \in N_{\delta}(0)$ for all $n \geq N_{1}$. Therefore $\left(1-r_{n}\right) v_{1} \ll \epsilon$. Also, $v_{n} \leq v_{1}$ and

$$
0<v_{n}-u_{n} \leq\left(1-r_{n}\right) v_{n} \leq\left(1-r_{n}\right) v_{1} \ll \epsilon
$$

By Remark 1.1, $\lim _{n \rightarrow \infty} u_{n}=\lim _{n \rightarrow \infty} v_{n}$. 
For all $n, p \geq 1$, applying the same argument, we have

$$
0<v_{n}-v_{n+p} \leq v_{n}-u_{n} \ll \epsilon
$$

Also,

$$
0<u_{n+p}-u_{n} \leq v_{n}-u_{n} \ll \epsilon \text {. }
$$

Hence, $\left\{u_{n}\right\}$ and $\left\{v_{n}\right\}$ are Cauchy sequences in $E$, then there exist $u^{*}, v^{*} \in E$ such that $u_{n} \rightarrow$ $u^{*}, v_{n} \rightarrow v^{*}(n \rightarrow \infty)$ and $u^{*}=v^{*}$. Write $x^{*}=u^{*}=v^{*}$.

It is easy to see $u_{0} \leq u_{n} \leq u^{*} \leq v_{n} \leq v_{0}$ for all $n=1,2, \ldots$ In addition, $S$ is closed, then $u^{*} \in\left[u_{n}, v_{n}\right] \cap S \subset\left[u_{0}, v_{0}\right] \cap S(n=0,1,2, \ldots)$.

Finally, by the mixed monotone property of $A$,

$$
u_{n-1} \leq A\left(u_{n}, v_{n}\right) \leq A\left(x^{*}, x^{*}\right) \leq A\left(u_{n}, v_{n}\right) \leq u_{n-1} \text {. }
$$

On taking limit on both sides of (11), when $n \rightarrow \infty$, we have $A\left(x^{*}, x^{*}\right)=x^{*}$. This means $x^{*}$ is a fixed point of $A$ in $\left[u_{0}, v_{0}\right] \cap S$.

Corollary 2.1 Let $P$ be a cone of $E$, let $S$ be a completely ordered closed subset of $E$ with $S_{0}=S \backslash\{\theta\} \subset \operatorname{int} P$ and let $\lambda S \subset S$ for all $\lambda \in[0,1]$. Let $u_{0}, v_{0} \in S_{0}, A: P \times P \rightarrow E$ satisfy

$$
\int_{y}^{t x} \phi d_{P} \leq \int_{t y}^{x} \phi d_{P}-\Psi\left(t, x, y, \int_{t y}^{x} \phi d_{P}\right)
$$

for all $(x, y) \in D \times D$, where $\Psi:[0,1) \times P \times P \times E \rightarrow E$ is an $\mathcal{L}^{\prime \prime}$-function, and let $\phi: P \rightarrow P$ be a non-vanishing, cone integrable mapping on each $[a, b] \subset P$ such that for each $\epsilon \gg 0$, $\int_{0}^{\epsilon} \phi d_{p} \gg 0$ and the mapping $\theta(x)=\int_{0}^{x} \phi d_{P}$ for $(x \geq 0)$ has a continuous inverse at zero. Also, $A\left(\left(\left[\theta, v_{0}\right] \cap S\right) \times\left(\left[\theta, v_{0}\right] \cap S\right)\right) \subset S$ satisfies the following conditions:

(I) there exists $r_{0}>0$ such that $u_{0} \geq r_{0} v_{0}$,

(II) $A\left(u_{0}, v_{0}\right) \ll u_{0} \ll v_{0} \ll A\left(v_{0}, u_{0}\right)$,

(III) for $u, v \in\left[u_{0}, v_{0}\right] \cap S$ with $A(u, v) \ll u \ll v$, there exists $u^{\prime} \in S$ such that $u \leq A\left(u^{\prime}, v\right) \ll u^{\prime} \ll v ;$ similarly, for $u, v \in\left[u_{0}, v_{0}\right] \cap S$ with $u \ll v \ll A(v, u)$, there exists $v^{\prime} \in S$ such that $u \ll v^{\prime} \ll A\left(v^{\prime}, u\right) \leq v$.

Then $A$ has at least one fixed point $x^{*} \in\left[u_{0}, v_{0}\right] \cap S$.

Proof Define

$$
A(x, y)=\int_{y}^{x} \phi d_{P}
$$

$A$ is a mixed monotone operator, and one can easily see that all conditions of Theorem 2.1 hold. Thus we obtain the desired result.

\section{$3 M_{3} R$ property}

In this section, we introduce a new fixed point theorem in the class of multi-valued mixed monotone operators. Due to this, the following definition is given. 
Definition 3.1 A mixed monotone operator $T: D \times D \rightarrow \mathcal{C}(E)$ is said to be a Mixed Monotone Multi-valued operator of Rhoades type $\left(\mathrm{M}_{3} \mathrm{R}\right.$ property for short $)$ if

$$
T(t x, y) \preceq T(x, t y)-\Psi(t, x, y, T(t x, y))
$$

for each $(x, y) \in D \times D$, where $\Psi:[0,1) \times P \times P \times E \rightarrow E$ is an $\mathcal{L}^{\prime \prime}$-function.

Theorem 3.1 Let $P$ be a cone of $E$, let $S$ be a completely ordered closed subset of $E$ with $S_{0}=$ $S \backslash\{\theta\} \subset \operatorname{int} P$ and let $\lambda S \subset S$ for all $\lambda \in[0,1]$. Let $u_{0}, v_{0} \in S_{0}, T: P \times P \rightarrow \mathcal{C}(E)$ be a mixed monotone multi-valued operator of Rhoades type with $T\left(\left(\left[\theta, v_{0}\right] \cap S\right) \times\left(\left[\theta, v_{0}\right] \cap S\right)\right) \subset S$ satisfying the following conditions:

(I) there exists $r_{0}>0$ such that $u_{0} \geq r_{0} v_{0}$,

(II) $T\left(u_{0}, v_{0}\right) \prec u_{0} \ll v_{0} \prec T\left(v_{0}, u_{0}\right)$,

(III) for $u, v \in\left[u_{0}, v_{0}\right] \cap S$ with $T(u, v) \prec u \ll v$, there exists $u^{\prime} \in S$ such that $u \preceq T\left(u^{\prime}, v\right) \prec u^{\prime} \ll v$; similarly, for $u, v \in\left[u_{0}, v_{0}\right] \cap S$ with $u \ll v \prec T(v, u)$, there exists $v^{\prime} \in S$ such that $u \ll v^{\prime} \prec T\left(v^{\prime}, u\right) \preceq v$.

Then $T$ has at least one fixed point $x^{*} \in\left[u_{0}, v_{0}\right] \cap S$.

Proof By the above condition (III), there exists $u_{1} \in S$ such that $u_{0} \preceq T\left(u_{1}, v_{0}\right) \prec u_{1} \ll$ $v_{0}$. Then there exists $v_{1} \in S$ such that $u_{1} \ll v_{1} \prec T\left(v_{1}, u_{1}\right) \preceq v_{0}$. Likewise, there exists $u_{2} \in S$ such that $u_{1} \preceq T\left(u_{2}, v_{1}\right) \prec u_{2} \ll v_{1}$. Then there exists $v_{2} \in S$ such that $u_{2} \ll v_{2} \prec$ $T\left(v_{2}, u_{2}\right) \leq v_{1}$. In general, there exists $u_{n} \in S$ such that $u_{n-1} \preceq T\left(u_{n}, v_{n-1}\right) \prec u_{n} \ll v_{n-1}$. Then there exists $v_{n} \in S$ such that $u_{n} \ll v_{n} \prec T\left(v_{n}, u_{n}\right) \preceq v_{n-1}(n=1,2, \ldots)$.

Take $r_{n}=\sup \left\{r \in(0,1): u_{n} \geq r v_{n}\right\}$, thus $0<r_{0}<r_{1}<\cdots<r_{n}<r_{n+1}<\cdots<1$, and $\lim _{n \rightarrow \infty} r_{n}=\sup \left\{r_{n}: n=0,1,2, \ldots\right\}=r^{*} \in(0,1]$. Since $r_{n+1}>r_{n}=\sup \left\{r \in(0,1): u_{n} \geq r v_{n}\right\}$, thus $u_{n} \nsucceq r_{n+1} v_{n}$. In addition, $S$ is completely ordered and $\lambda S \subset S$ for all $\lambda \in[0,1]$, then $u_{n}<r_{n+1} v_{n}$. Now, one can prove $r^{*}=1$. Otherwise, $r^{*} \in(0,1)$. We claim

$$
T\left(u_{n+1}, v_{n+1}\right) \preceq T\left(\left(1 / r^{\prime \prime}\right) u_{n+1}, r^{\prime \prime} v_{n+1}\right) .
$$

Suppose that $x \in T\left(u_{n+1}, v_{n+1}\right)$ is arbitrary. We have $u_{n+1} \leq\left(1 / r^{*}\right) u_{n+1}$. If $x_{1}=u_{n+1}, x_{2}=$ $\left(1 / r^{*}\right) u_{n+1}$ and $y=v_{n+1}$, then by $\left(\mathrm{A}_{1}\right)$ of Definition 1.5, there exists $z \in T\left(\left(1 / r^{*}\right) u_{n+1}, v_{n+1}\right)$ such that $x \leq z$. Thus, $T\left(u_{n+1}, v_{n+1}\right) \preceq T\left(\left(1 / r^{*}\right) u_{n+1}, v_{n+1}\right)$.

Also, if $y_{1}=r^{*} v_{n+1}, y_{2}=v_{n+1}$ and $x=\left(1 / r^{*}\right) u_{n+1}$, then for $w \in T\left(\left(1 / r^{*}\right) u_{n+1}, r^{*} v_{n+1}\right)$, there exists $h \in T\left(\left(1 / r^{*}\right) u_{n+1}, v_{n+1}\right)$ such that $w \geq h$. It means that

$$
T\left(\left(1 / r^{*}\right) u_{n+1}, v_{n+1}\right) \preceq T\left(\left(1 / r^{*}\right) u_{n+1}, r^{*} v_{n+1}\right) .
$$

Thus,

$$
\begin{aligned}
T\left(u_{n+1}, v_{n+1}\right) & \preceq T\left(\left(1 / r^{*}\right) u_{n+1}, r^{*} v_{n+1}\right) \\
& \preceq T\left(u_{n+1}, v_{n+1}\right)-\Psi\left(\frac{1}{r^{*}}, u_{n+1}, r^{*} v_{n+1}, T\left(u_{n+1}, v_{n+1}\right)\right) \\
& \prec T\left(u_{n+1}, v_{n+1}\right),
\end{aligned}
$$

and this is a contradiction. Therefore, $r^{*}=1$. Let $\epsilon \gg 0$ be given. Choose $\delta>0$ such that $\epsilon+N_{\delta}(0) \subseteq P$, where $N_{\delta}(0)=\{y \in E:\|y\|<\delta\}$. Since $r_{n} \rightarrow 1$, one can choose a natural 
Figure $1 A\left(u_{0}, v_{0}\right) \ll u_{0} \ll u_{1} \ll \cdots \ll u_{n} \ll$ $\cdots \ll 1 \ll \cdots \ll v_{n} \ll \cdots \ll v_{1} \ll v_{0} \ll A\left(v_{0}, u_{0}\right)$.

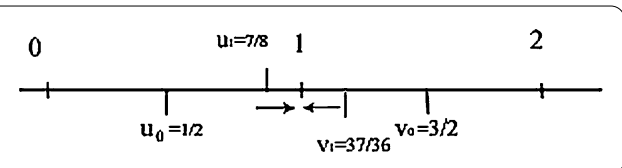

number $N_{1}$ such that $\left(1-r_{n}\right) v_{1} \in N_{\delta}(0)$ for all $n \geq N_{1}$. Therefore $\left(1-r_{n}\right) v_{1} \ll \epsilon$. Also, $v_{n} \leq v_{1}$ and

$$
0<v_{n}-u_{n} \leq\left(1-r_{n}\right) v_{n} \leq\left(1-r_{n}\right) v_{1} \ll \epsilon .
$$

By Remark 1.1, $\lim _{n \rightarrow \infty} u_{n}=\lim _{n \rightarrow \infty} v_{n}$.

For all $n, p \geq 1$, applying the same argument, we have

$$
0<v_{n}-v_{n+p} \leq v_{n}-u_{n} \ll \epsilon
$$

Also,

$$
0<u_{n+p}-u_{n} \leq v_{n}-u_{n} \ll \epsilon .
$$

Hence, $\left\{u_{n}\right\}$ and $\left\{v_{n}\right\}$ are Cauchy sequences in $E$, then there exist $u^{*}, v^{\prime \prime} \in E$ such that $u_{n} \rightarrow$ $u^{*}, v_{n} \rightarrow v^{*}(n \rightarrow \infty)$ and $u^{*}=v^{*}$. Write $x^{*}=u^{*}=v^{*}$.

It is easy to see that $u_{n} \preceq T\left(u_{n+1}, v_{n+1}\right) \preceq T\left(x^{\prime \prime}, x^{\prime \prime}\right) \preceq T\left(v_{n+1}, u_{n+1}\right) \preceq v_{n}$ for all $n=1,2, \ldots$. Thus, there exists $z_{n} \in T\left(x^{*}, x^{*}\right)$ such that $u_{n} \leq z_{n} \leq v_{n}$. By taking limit on both sides of (17),

$$
0<z_{n}-u_{n} \leq\left(1-r_{n}\right) v_{n} \leq\left(1-r_{n}\right) v_{1} \ll \epsilon .
$$

So, $z_{n} \rightarrow x^{*}$. Since $T$ has closed values, then $x^{*} \in T\left(x^{*}, x^{*}\right)$ and

$$
x^{*} \in\left[u_{n}, v_{n}\right] \cap S \subset\left[u_{0}, v_{0}\right] \cap S .
$$

Remark 3.1 One can see easily that Theorem 2.1 should be included as a corollary of Theorem 3.1.

Example 3.1 Let $E=\mathrm{R}, P=[0,+\infty)$ and $S=P$. Then $S_{0}=\operatorname{int}(P)=(0,+\infty)$.

Define $A:[0,+\infty) \times[0,+\infty) \rightarrow \mathrm{R}$ as

$$
A(x, y)= \begin{cases}\frac{x}{y}, & (x, y) \neq(0,0) \\ 0, & (x, y)=(0,0)\end{cases}
$$

$A$ is a mixed monotone operator. Now suppose that $\Psi:[0,1) \times P \times P \times E \rightarrow E$ is as $\Psi(t, x, y, s)=\left(1-t^{2}\right) s$. Then $\Psi$ is an $\mathcal{L}^{\prime \prime}$-function. Moreover,

$$
A(t x, y) \leq A(x, t y)-\Psi(t, x, y, A(x, t y))
$$

for each $x, y \in S_{0}$. Also, by taking $u_{0}=\frac{1}{2}, v_{0}=\frac{3}{2}$ and $r_{0}=\frac{1}{4}$, we have

(I) $u_{0} \geq r_{0} v_{0}$ 
(II) $A\left(u_{0}, v_{0}\right)=\frac{1}{3} \ll u_{0} \ll v_{0} \ll A\left(v_{0}, u_{0}\right)=3$,

(III) for $u, v \in\left[u_{0}, v_{0}\right] \cap S$ with $A(u, v) \ll u \ll v$, there exists $u^{\prime} \in S$ such that $u \leq A\left(u^{\prime}, v\right) \ll u^{\prime} \ll v$; similarly, for $u, v \in\left[u_{0}, v_{0}\right] \cap S$ with $u \ll v \ll A(v, u)$, there exists $v^{\prime} \in S$ such that $u \ll v^{\prime} \ll A\left(v^{\prime}, u\right) \leq v$.

For further explanation on (III), since $A\left(u_{0}, v_{0}\right)=\frac{1}{3} \ll u_{0} \ll v_{0}$, by (III) there exists $u_{1} \in S$ such that $u_{0} \ll A\left(u_{1}, v_{0}\right) \ll u_{1} \ll v_{0}$. It means that $\frac{1}{2} \ll \frac{u_{1}}{\frac{3}{2}} \ll u_{1} \ll \frac{3}{2}$. Thus $u_{1}$ must be greater than $\frac{3}{4}$. Therefore we can set $u_{1}=\frac{\frac{3}{4}+1}{2}$. Similarly, since $\frac{7}{8}=u_{1} \ll v_{0}=\frac{3}{2} \ll$ $A\left(v_{0}, u_{1}\right)=\frac{12}{7}$, thus by (III) there exists $v_{1} \in S$ such that $u_{1} \ll v_{1} \ll A\left(v_{1}, u_{1}\right) \leq v_{0}$. It means that $v_{1}$ must be less than $\frac{21}{16}$. We can set $v_{1}=\frac{\frac{21}{16}+1}{2}$. By the continuity of such ways, we can consider the following reflexive sequences:

$$
u_{0}=\frac{1}{2}, \quad v_{0}=\frac{3}{2}, \quad u_{n}=\frac{u_{n-1} v_{n-1}+1}{2} \quad \text { and } \quad v_{n}=\frac{v_{n-1} u_{n}+1}{2}
$$

which satisfy (I), (II) and (III) (see Figure 1). Moreover, $u_{n} \rightarrow 1$ and $v_{n} \rightarrow 1$ and $A(1,1)=1$.

\section{Application}

The following result is given by Zhang [6] and is obtained by our main result.

Corollary 4.1 Let $P$ be a normal cone of $E$, let $S$ be a completely ordered closed subset of $E$ with $S_{0}=S \backslash\{\theta\} \subset$ int $P$ and let $\lambda S \subset S$ for all $\lambda \in[0,1]$. Let $u_{0}, v_{0} \in S_{0}, A: P \times P \rightarrow E$ be a mixed monotone operator with $A\left(\left(\left[\theta, v_{0}\right] \cap S\right) \times\left(\left[\theta, v_{0}\right] \cap S\right)\right) \subset S$ and $A\left(u_{0}, v_{0}\right) \ll u_{0} \ll$ $v_{0} \ll A\left(v_{0}, u_{0}\right)$. Assume that there exists a function $\phi:(0,1) \times\left(\left[u_{0}, v_{0}\right] \cap S\right) \times\left(\left[u_{0}, v_{0}\right] \cap\right.$ $S) \rightarrow(0,+\infty)$ such that $A(t x, y) \leq \phi(t, x, y) A(x, t y)$, where $0<\phi(t, x, x)<t$ for all $(t, x, y) \in$ $(0,1) \times\left(\left[u_{0}, v_{0}\right] \cap S\right) \times\left(\left[u_{0}, v_{0}\right] \cap S\right)$. Suppose that

(I) for $u, v \in\left[u_{0}, v_{0}\right] \cap S$ with $A(u, v) \ll u \ll v$, there exists $u^{\prime} \in S$ such that $u \leq A\left(u^{\prime}, v\right) \ll u^{\prime} \ll v ;$ similarly, for $u, v \in\left[u_{0}, v_{0}\right] \cap S$ with $u \ll v \ll A(v, u)$, there exists $v^{\prime} \in S$ such that $u \ll v^{\prime} \ll A\left(v^{\prime}, u\right) \leq v$.

(II) there exists an element $w_{0} \in\left[u_{0}, v_{0}\right] \cap S$ such that $\phi(t, x, x) \leq \phi\left(t, w_{0}, w_{0}\right)$ for all $(t, x) \in(0,1) \times\left(\left[u_{0}, v_{0}\right] \cap S\right)$, and $\lim _{s \rightarrow t^{-}} \phi\left(s, w_{0}, w_{0}\right)<t$ for all $t \in(0,1)$.

Then $A$ has at least one fixed point $x^{*} \in\left[u_{0}, v_{0}\right] \cap S$.

Proof Set $\Psi(t, x, y, z)=(1-\phi(t, x, y)) z$. Then $\Psi$ is an $\mathcal{L}^{\prime \prime}$-function, and we have

$$
A(t x, y) \leq \phi(t, x, y) A(x, t y)=A(x, t y)-\Psi(t, x, y, A(x, t y)) .
$$

Thus, by Theorem 2.1 the desired result is obtained.

\section{Competing interests}

The author declares that they have no competing interests.

Received: 11 May 2012 Accepted: 1 March 2013 Published: 28 March 2013

\section{References}

1. Guo, DJ, Lakshmikantham, V: Coupled fixed points of nonlinear operators with applications. Nonlinear Anal. TMA 11, 623-632 (1987)

2. Guo, DJ: Fixed points of mixed monotone operators with applications. Appl. Anal. 31, $215-224$ (1988)

3. Hong, S: Fixed points for mixed monotone multivalued operators in Banach spaces with applications. J. Math. Anal. Appl. 337, 333-342 (2008) 
4. Wu, Y: New fixed point theorems and applications of mixed monotone operator. J. Math. Anal. Appl. 341, 883-893 (2008)

5. Xu, S, Jia, B: Fixed-point theorems of $\phi$ concave- $\psi$ convex mixed monotone operators and applications. J. Math. Anal. Appl. 295, 645-657 (2004)

6. Zhang, M: Fixed point theorems of $\phi$ concave- $\psi$ convex mixed monotone operators and applications. J. Math. Anal. Appl. 339, 970-981 (2008)

7. Zhang, Z, Wang, K: On fixed point theorems of mixed monotone operators and applications. Nonlinear Anal. TMA 70, 3279-3284 (2009)

8. Zhao, Z: Existence and uniqueness of fixed points for some mixed monotone operators. Nonlinear Anal. TMA 73, $1481-1490$ (2010)

9. Rhoades, BE: Some theorems on weakly contractive maps. Proceedings of the Third World Congress of Nonlinear Analysis, part 4 (Catania, 2000). Nonlinear Anal. TMA 47, 2683-2693 (2001)

10. Khojasteh, F, Razani, A: Fixed point theorems for single-valued and multi-valued mixed monotone operators of Meir-Keeler type. J. Nonlinear Convex Anal. 14(2) (2013, to appear)

11. Khojasteh, F, Goodarzi, Z, Razani, A: Some fixed point theorems of integral type contraction in cone metric spaces. Fixed Point Theory Appl. (2010). doi:10.1155/2010/189684

12. Khojasteh, F, Razani, A, Moradi, S: A fixed point of generalized $T_{F}$-contraction mappings in cone metric spaces. Fixed Point Theory Appl. (2011). doi:10.1186/1687-1812-2011-14

13. Arandelovic, I, Kadelburg, Z, Radenovic, S: Boyd-Wong-type common fixed point results in cone metric spaces. Appl. Math. Comput. 217, 7167-7171 (2011)

doi:10.1186/1687-1812-2013-73

Cite this article as: Khojasteh: Some new results for single-valued and multi-valued mixed monotone operators of Rhoades type. Fixed Point Theory and Applications 2013 2013:73.

\section{Submit your manuscript to a SpringerOpen ${ }^{\circ}$ journal and benefit from:}

- Convenient online submission

- Rigorous peer review

- Immediate publication on acceptance

- Open access: articles freely available online

- High visibility within the field

- Retaining the copyright to your article 